\title{
Tocilizumab Effectiveness After Switching from Intravenous to Subcutaneous Route in Patients with Rheumatoid Arthritis: The RoSwitch Study
}

\author{
Jean Darloy · Nicolas Segaud · Jean-Hugues Salmon · Jean-Paul Eschard • Vincent Goëb • \\ Xavier Deprez • Marie-Hélène Guyot • Eric Houvenagel • Nicolas Lecuyer • Laurent Marguerie • \\ Samuel Gally · David Pau · Isabelle Idier · Guy Baudens · René-Marc Flipo
}

Received: October 31, 2018 / Published online: January 10, 2019

(C) The Author(s) 2019

\section{ABSTRACT}

Introduction: The main objective of this work was to assess the maintenance of effectiveness of subcutaneous tocilizumab 6 months after switching from intravenous to subcutaneous formulation in patients with rheumatoid arthritis (RA) in a real-world setting. Secondary objectives aimed to describe the characteristics

Enhanced digital features To view enhanced digital features for this article go to https://doi.org/10.6084/ m9.figshare.7485626.

Electronic supplementary material The online version of this article (https://doi.org/10.1007/s40744018-0138-y) contains supplementary material, which is available to authorized users.

J. Darloy $(\bowtie) \cdot$ N. Segaud · R.-M. Flipo

Rheumatology Department, Hôpital Roger Salengro,

University Hospital of Lille, Lille, France

e-mail: jeandarloy@gmail.com

J.-H. Salmon · J.-P. Eschard

Rheumatology Department, Hôpital Maison

Blanche, University Hospital of Reims, Reims,

France

V. Goëb

Rheumatology Department, Hôpital Nord,

University Hospital of Amiens-Picardie, Amiens,

France

X. Deprez · M.-H. Guyot

Rheumatology Department, Hôpital Jean Bernard, Hospital of Valenciennes, Valenciennes, France of patients and disease, the effectiveness at 12 months after switching, the therapeutic maintenance, and to search for predictive factors of switching.

Methods: We analyzed all the RA patients of the shared medical file "RIC Nord de France", treated with tocilizumab, switching or not from intravenous to subcutaneous tocilizumab, between April 2015 and January 2016. The primary effectiveness endpoint was the proportion of patients remaining in their DAS28-ESR category remission/low disease activity (LDA) or moving to an inferior DAS28-ESR category at 6 months. Since RoSwitch was an observational study, without randomization, a propensity score was built in a sensitivity analysis to balance on RA and patients' characteristics at inclusion between switching and no-switching groups.

\section{M.-H. Guyot}

Rheumatology Department, Hôpital Victor Provo, Hospital of Roubaix, Roubaix, France

E. Houvenagel

Rheumatology Department, Hôpital Saint Philibert, Hospital of Lomme, Lomme, France

N. Lecuyer

Rheumatology, Medical office, Saint-Quentin, France

L. Marguerie

Rheumatology Department, Institut François Calot, Berck-Sur-Mer, France 
Results: An improvement of initial DAS28-ESR category or maintenance in DAS28-ESR remission/LDA at 6 months was shown in 203 of the 285 patients with an evaluation for the primary criterion $(71.2 \%, 95 \%$ CI [65.6-76.4\%]) without differences between groups $(73.3 \%, 95 \% \mathrm{CI}$ [63.0-82.1\%] vs. 70.3\%, 95\% CI [63.3-76.6\%]). The RoSwitch study showed the maintenance of effectiveness at 6 and 12 months. Similar therapeutic maintenance rates were observed for switch and no-switch patients. No clinical factor was associated with the switch in patients in remission/LDA at inclusion.

Conclusions: The RoSwitch study showed the maintenance of effectiveness at 6 months in RA patients switching from intravenous (IV) to subcutaneous (SC) tocilizumab.

Funding: Roche SAS and Chugai Pharma France.

Keywords: Administration route; Rheumatoid arthritis; Subcutaneous; Switch; Tocilizumab

\section{INTRODUCTION}

Rheumatoid arthritis (RA) is a multifactorial chronic inflammatory rheumatism of unknown etiology. Affecting $0.3-0.5 \%$ of the population in France [1], its evolution is associated with progressive joint destruction and increased cardiovascular risk [2], with significant functional and medico-economic impacts [3]. Interleukin-6 (IL$6)$, whose concentration in serum and synovial fluid is particularly high in patients with RA, participates in inflammatory reactions [4].

Since 2009, in France, the therapeutic arsenal of RA has expanded with the emergence of new biomedicines, including tocilizumab (TCZ), which is the first humanized monoclonal

S. Gally · D. Pau

Clinical Operations, Roche SAS, Boulogne-

Billancourt, France

I. Idier

Medical Department, Chugai Pharma France, Paris

La Défense, France

G. Baudens

Rheumatology, Medical office, Valenciennes, France antibody that specifically inhibits both soluble and membrane-bound IL-6 receptors. This treatment was delivered intravenously (IV) since 2009, then subcutaneously (SC) from 2015 in France. The development of TCZ included seven large international double-blind phase III clinical trials conducted in patients with moderate-to-severe active RA and inadequate response to csDMARD [5-11], two of which assessed the effectiveness and the safety of the $S C$ route $[10,11]$.

However, the study of the switch from IV to SC TCZ, with also an analysis of patients remaining in IV, has not been evaluated to date in the long term in a large real-world population of RA patients. In this context, this database analysis was performed to improve the knowledge of current practice of TCZ administration in patients with RA. The main objective was to assess the maintenance of effectiveness of SC TCZ 6 months after switching from IV to SC formulation in patients with RA in a realworld setting. Secondary objectives aimed to describe the characteristics of patients and disease, the effectiveness of SC TCZ 12 months after switching, the therapeutic maintenance, and to search for predictive factors of switching.

\section{METHODS}

\section{Patients}

RoSwitch was a 1-year, non-interventional secondary data use study, based on a French regional database, the "RIC Nord de France" Electronic Medical Records (EMR), whose aim is to focus exchange information on treated patients suffering from chronic inflammatory rheumatisms between office-based and hospital clinicians. The present database analysis study was carried out on a subpopulation from the RIC Nord de France EMR database. In accordance with French law regarding non-interventional studies, RoSwitch protocol was approved on April 7, 2016 by the Consultative Committee on Information Processing for Research in the Field of Health and was validated on August 1, 2016 by the Independent Administrative Authority Protecting Privacy 
and Personal Data. All patients were informed about the course of the study before enrolment. This study was performed in accordance with the Helsinki Declaration of 1964 and its later amendments.

Once they had given their informed consent, we extracted all the RA patients aged more than 18 years old treated with IV or SC TCZ between April 30, 2015 (first day of availability of SC TCZ in France) and January 15, 2016.

Initially, we defined two analysis populations, both with previous duration of IV $\mathrm{TCZ} \geq 3$ months: the main population, in which patients had a DAS28 value within 3 months before or at inclusion, and the stable population, in which patients had 2 DAS28 within 3 months before or at inclusion in the same category.

In parallel, two groups were defined on the basis of a shared decision between the patients and their rheumatologists depending on the route of the treatment: a group comprising patients switching from IV to SC TCZ (162 mg every week), the switch group, and a group with patients continuing IV TCZ, the no-switch group. In the no-switch group at inclusion, 162 patients were at $8 \mathrm{mg} / \mathrm{kg}$ every 4 weeks, 16 were at $6 \mathrm{mg} / \mathrm{kg}$ every 4 weeks, and 42 were at $4 \mathrm{mg} /$ $\mathrm{kg}$ every 4 weeks. For switch patients, the inclusion date was defined as the switch date between April 30, 2015 and January 15, 2016. For no-switch patients, the inclusion date was defined as the first injection of IV TCZ in the same period.

\section{Outcomes and Assessments}

At inclusion, 6 and 12 months, the DAS28-ESR score was extracted from the database, and patients were classified by activity category as follows: remission (DAS28-ESR $\leq 2.6$ ), low disease activity (LDA) $(2.6<$ DAS28-ESR $\leq 3.2)$, moderate disease activity (MDA) $(3.2<$ DAS28-ESR $\leq 5.1)$, high disease activity (HDA) (DAS28-ESR > 5.1).

At inclusion, the following variables were extracted: sociodemographics data, comorbidities and concomitant diseases, RA history and previous treatments, clinical and laboratory data related to RA, and use of TCZ and concomitant RA treatments.
At 6 and 12 months, we collected the following variables: clinical and erythrocyte sedimentation rate (ESR) data related to RA, and use of TCZ and concomitant RA treatments.

Data from a patient self-reported questionnaire, notably on the reasons for the switch of administration route or not and on perceptions on SC injections, were also extracted at inclusion and 6 months.

The primary criterion was the proportion of patients remaining in their DAS28-ESR remission/LDA inclusion category or moving to an inferior category at 6 months after the switch from IV to SC. Secondary criteria were DAS28ESR, TCZ maintenance rate up to 12 months and predictive factors of the switch.

\section{Statistical Methods}

Summary descriptive statistics were provided for each variable described above. The proportion of patients remaining in their DAS28-ESR remission/LDA inclusion category or moving to an inferior category at 6 months was provided with its 95\% confidence interval (95\% CI), using the Clopper-Pearson exact method. The permanent discontinuation of treatment before 6 months (without change of administration route) was considered as treatment failure. The same analysis was carried out at 12 months. The Kaplan-Meier method was used for therapeutic maintenance. The comparison between switch and no-switch patients was tested using the logrank test and Wilcoxon test over the 6- and 12-month periods. Logistic methods were used for predictive factor research of switch. To balance the two groups of patients according to the baseline criteria, a propensity score was built using a multivariate logistic regression model. Selected variables and relevant clinical prognostic factors of RA (smoking status, familial history of RA, rheumatoid factor [RF], anticitrullinated protein antibody [ACPA], the presence of at least one joint erosion, and daily dose of glucocorticoids) were considered as covariates. Variables were selected using backward selection with a retain threshold of 0.20 . Weighted method (inverse probability of treatment weight, IPTW) was used and the 
proportion of the primary criterion was provided with its $95 \% \mathrm{CI}$, using the bootstrap method and the 2.5th and 97.5th percentiles of the distribution obtained. All statistical analyses were performed using SAS $^{\circledR}$ version 9.4 software (SAS Institute Inc., Cary, NC, USA).

Exploratory analysis, using data mining techniques such as decision trees and random forests, were used to search for factors related to the DAS28-ESR evolution between inclusion and 6 months, with additional factors of interest (body mass index [BMI], smoker status, combination of tocilizumab with csDMARD) compared to previously mentioned logistic methods.

Although safety analysis was not planned for this study, adverse events were collected in all RA TCZ-treated patients in the EMR. Separate descriptive safety analysis was performed from a distinct dataset of patients during the concerned period. Inefficiency as well as remission were reported as events and defined as per investigators judgment.

\section{RESULTS}

\section{Patient Disposition and Baseline Characteristics}

Amongst the 328 patients from the extracted database (Fig. 1), 14 patients were excluded from the main population, bringing this analysis population to 314 patients, including 94 switch patients and 220 no-switch patients. There were 68 switch patients and 136 noswitch patients in the stable population.

Table 1 shows the baseline characteristics in the main population. Similar results were observed in the stable population.

\section{Maintenance of Effectiveness}

In the main population, an improvement of initial DAS28-ESR category or maintenance in DAS28-ESR remission/LDA at 6 months was shown in 203 of the 285 patients with an evaluation for the primary criterion $(71.2 \%, 95 \% \mathrm{CI}$ [65.6-76.4\%]) without differences between groups (73.3\%, 95\% CI [63.0-82.1\%] vs. 70.3\%,
95\% CI [63.3-76.6\%]) (Table 2). In the stable population, this proportion was slightly higher $(75.0 \%$, CI 95\% [68.1-81.1\%]) and similarly, no differences between groups were observed $(75.4 \%, 95 \%$ CI [63.1-85.2\%] vs. $74.8 \%, 95 \%$ CI [66.0-82.3\%]). Using the IPTW for balancing on RA and patients characteristics between the switch and no-switch groups, a similar proportion of patients with improvement of DAS28-ESR category or maintenance of a remission/LDA status was observed in the main population compared to results from the primary analysis (72.7 vs. $71.2 \%$ ), as well as for switch and no-switch patients $(72.8$ and $72.5 \%$ vs. 73.3 and $70.3 \%$, respectively). Similar results were observed in the stable population.

In the main population, for switch patients, improvement of initial DAS28-ESR category or maintenance in DAS28-ESR remission/LDA at 6 months was similar in monotherapy patients and patients receiving TCZ combined with a csDMARD (72.7\%, 95\% CI [57.2-85.0\%] vs. 73.3\%, 95\% CI [58.1-85.4\%]).

When using the data mining techniques, neither BMI, smoking status, nor TCZ used as monotherapy were significantly related to the DAS28-ESR change between inclusion and 6 months.

At 12 months, an improvement of initial DAS28-ESR category or maintenance in DAS28ESR remission/LDA was shown in 181 patients (61.4\%, 95\% CI [55.5-66.9\%]) of the main population with a favorable trend observed in switch patients compared to no-switch patients (69.2\%, 95\% CI [58.7-78.5\%] vs. 57.8\%, 95\% CI [50.8-64.7\%]) (Table 3). Using the IPTW score in the main population, it was observed a slightly higher proportion of patients with DAS28-ESR improvement or maintenance of a remission/LDA status at 12 months than in the primary analysis. Similarly, a higher proportion of switch patients presented with favorable outcomes.

DAS28-ESR improvement or maintenance of remission/LDA status at 6 and 12 months according to the DAS28-ESR category at inclusion is shown in Tables S1 and S2.

In the main population, the mean DAS28ESR score slightly decreased from inclusion to 12 months in switch and no-switch patients 


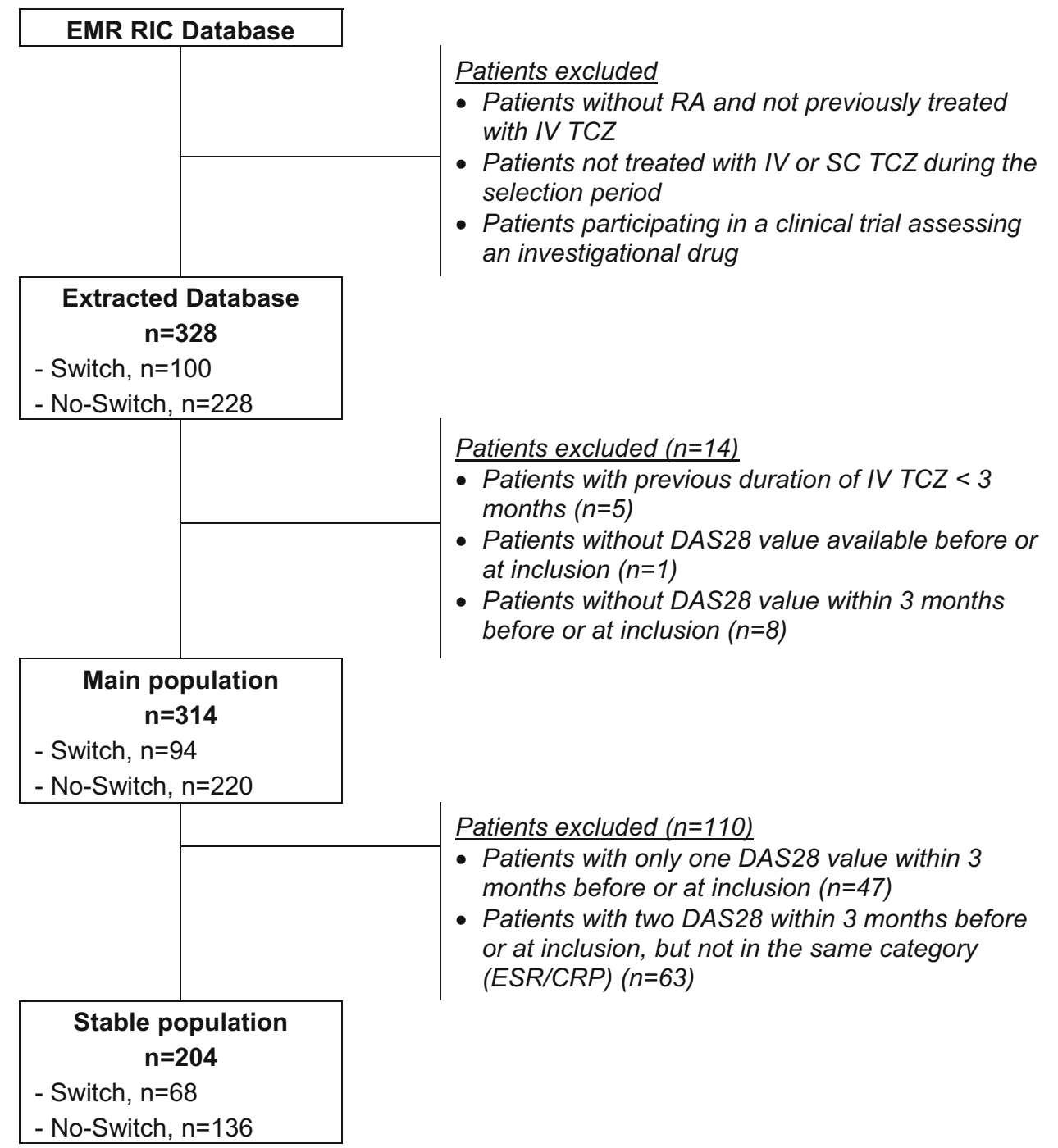

Fig. 1 Flowchart-analysis populations from the extracted database. CRP C-reactive protein, DAS28 disease activity score in 28 joints, EMR electronic medical records, ESR erythrocyte sedimentation rate, $I V$ intravenous, $R A$

(from $2.14 \pm 1.14$ to $1.74 \pm 0.91$ and from $2.95 \pm 1.64$ to $2.48 \pm 1.31$, respectively) (Fig. 2). The mean change from inclusion in DAS28-ESR value was limited with slight differences between patient groups: $-0.22 \pm 1.31$ in switch patients and $-0.38 \pm 1.65$ in no-switch patients at 6 months, $-0.43 \pm 1.13$ in switch patients and $-0.30 \pm 1.75$ in no-switch patients at 12 months. rheumatoid arthritis, RIC "rhumatismes inflammatoires chroniques" chronic inflammatory rheumatism, $S C$ subcutaneous, $T C Z$ tocilizumab

\section{Therapeutic Maintenance}

In the main population, treatment maintenance at 6 months in switch and no-switch patients was observed in $85.1 \% \quad(95 \% \quad \mathrm{CI}$ [76.2-90.9\%]) and 91.8\% (95\% CI [87.3-94.8\%]) of patients, respectively (Fig. 3). At 12 months, these respective proportions were $77.7 \%$ (95\% CI $\quad[67.8-84.8 \%])$ and $80.0 \% \quad(95 \% \quad$ CI [74.1-84.7\%]) (Fig. 3). No significant difference was found between groups at 6 months 
Table 1 Patients' baseline characteristics of the main population

\begin{tabular}{|c|c|c|c|}
\hline & All & Switch & No-switch \\
\hline \multicolumn{4}{|c|}{ Demographics data and smoking status } \\
\hline \multicolumn{4}{|l|}{ Gender } \\
\hline$n(\%)$ & 314 & 94 & 220 \\
\hline Female & $244(77.7)$ & $71(75.5)$ & $173(78.6)$ \\
\hline \multicolumn{4}{|l|}{ Age } \\
\hline$n(\%)$ & 314 & 94 & 220 \\
\hline $21-50$ years & $72(22.9)$ & $21(22.3)$ & $51(23.2)$ \\
\hline $51-70$ years & $176(56.0)$ & $51(54.2)$ & $125(56.8)$ \\
\hline $71-90$ years & $66(21.0)$ & $22(23.4)$ & $44(20.0)$ \\
\hline \multicolumn{4}{|l|}{ BMI $\left(\mathrm{kg} / \mathrm{m}^{2}\right)$} \\
\hline$n$ & 309 & 93 & 216 \\
\hline Mean (SD) & $27.5(6.4)$ & $27.1(5.8)$ & $27.7(6.7)$ \\
\hline \multicolumn{4}{|l|}{ Body weight (kg) } \\
\hline$n$ & 313 & 94 & 219 \\
\hline Mean (SD) & $74.67(17.91)$ & $73.88(16.55)$ & $75.01(18.49)$ \\
\hline \multicolumn{4}{|l|}{ Smoking status } \\
\hline$n(\%)$ & 287 & 87 & 200 \\
\hline Current smoker & $55(19.2)$ & $21(24.1)$ & $34(17.0)$ \\
\hline Never smoked & $185(64.5)$ & $54(62.1)$ & $131(65.5)$ \\
\hline Ever smoking & $47(16.4)$ & $12(13.8)$ & $35(17.5)$ \\
\hline \multicolumn{4}{|l|}{ RA bistory } \\
\hline \multicolumn{4}{|c|}{ Time to 1 st diagnosis (years) } \\
\hline$n$ & 314 & 94 & 220 \\
\hline Mean (SD) & $14.9(9.2)$ & $15.0(9.1)$ & $14.9(9.2)$ \\
\hline \multicolumn{4}{|l|}{$\mathrm{RF}$ or $\mathrm{ACPA}$} \\
\hline$n(\%)$ & 308 & 89 & 219 \\
\hline Positive & $273(88.6)$ & $76(85.4)$ & $197(90.0)$ \\
\hline \multicolumn{4}{|l|}{ Erosive RA } \\
\hline$n(\%)$ & 314 & 94 & 220 \\
\hline Yes & $232(73.9)$ & $72(76.6)$ & $160(72.7)$ \\
\hline \multicolumn{4}{|c|}{ Rheumatoid nodules } \\
\hline$n(\%)$ & 314 & 94 & 220 \\
\hline Yes & $30(9.6)$ & $15(16.0)$ & $15(6.8)$ \\
\hline
\end{tabular}


Table 1 continued

All Switch

No-switch

Previous $R A$ treatments

$\geq 1 \mathrm{csDMARD}$

$n(\%)$

313

Yes

$240(76.7)$

93

220

76 (81.7)

$164(74.5)$

Number of prior bDMARDs

$n(\%)$
1
2
$\geq 3$

Ongoing $R A$ treatments

MTX

$n(\%)$

Yes

Dose of MTX (mg/week)

n

Mean (SD)

Corticosteroids

$n(\%)$

Yes

Corticosteroids (mg/day)

n

Mean (SD)

IV TCZ duration (months)

n

Mean (SD)

Dose of IV TCZ $(\mathrm{mg} / \mathrm{kg})$

n

Mean (SD)

Disease activity at inclusion

DAS28-ESR

n

Mean (SD)
313

85 (27.2)

95 (30.4)

72 (23.0)

313

143 (45.7)

140

$13.0(4.3)$

312

129 (41.2)

128

$6.4(5.0)$

314

$29.2(22.7)$

314

7.1 (1.6)

314

2.7 (1.6)
93

220

23 (24.7)

62 (28.2)

28 (30.1)

67 (30.5)

24 (25.8)

48 (21.8)

93

220

41 (44.1)

$102(46.4)$

41

99

$12.9(3.2)$

$13.1(4.7)$

93

220

39 (41.9)

$90(40.9)$

38

90

$6.7(7.4)$

$6.3(3.7)$

94

220

$35.0(23.1)$

$26.8(22.1)$

94

220

$7.2(1.5)$

7.0 (1.7) 
Table 1 continued

\begin{tabular}{llll}
\hline & All & Switch & No-switch \\
\hline DAS28-ESR in categories & & & \\
$n(\%)$ & 314 & 94 & 220 \\
$\leq 3.2$ & $208(66.2)$ & $77(81.9)$ & $131(59.5)$ \\
{$[3.2-5.1]$} & $80(25.5)$ & $17(18.1)$ & $63(28.6)$ \\
$>5.1$ & $26(8.3)$ & 0 & $26(11.8)$ \\
\hline
\end{tabular}

$A C P A$ anti-citrullinated protein antibody, bDMARDs biological disease-modifying antirheumatic drugs, $B M I$ body mass index, $c s D M A R D$ conventional synthetic disease-modifying antirheumatic drug, DAS28-ESR disease activity score in 28 joints using the erythrocyte sedimentation rate, $I V$ intravenous, $M T X$ methotrexate, $R A$ rheumatoid arthritis, $R F$ rheumatoid factor, $T C Z$ tocilizumab

Table 2 DAS28-ESR improvement or maintenance of remission/LDA status at 6 months

\begin{tabular}{|c|c|c|c|c|}
\hline & \multicolumn{2}{|c|}{ Main population $(n=314)$} & \multicolumn{2}{|c|}{ Stable population $(n=204)$} \\
\hline & Switch $(n=94)$ & No-switch $(n=220)$ & Switch $(n=68)$ & No-switch $(n=136)$ \\
\hline \multicolumn{5}{|c|}{ Without propensity score } \\
\hline$n$ & 90 & 195 & 65 & 119 \\
\hline Missing & 4 & 25 & 3 & 17 \\
\hline No & $24(26.7 \%)$ & $58(29.7 \%)$ & $16(24.6 \%)$ & $30(25.2 \%)$ \\
\hline $95 \% \mathrm{CI}$ & {$[17.9-37.0 \%]$} & {$[23.4-36.7 \%]$} & {$[14.8-36.9 \%]$} & {$[17.7-34.0 \%]$} \\
\hline Yes & $66(73.3 \%)$ & $137(70.3 \%)$ & $49(75.4 \%)$ & $89(74.8 \%)$ \\
\hline $95 \% \mathrm{CI}$ & {$[63.0-82.1 \%]$} & {$[63.3-76.6 \%]$} & {$[63.1-85.2 \%]$} & {$[66.0-82.3 \%]$} \\
\hline \multicolumn{5}{|c|}{ With propensity score } \\
\hline$n$ & 149.0 & 141.2 & 94.7 & 88.1 \\
\hline No & $40.5(27.2 \%)$ & $38.8(27.5 \%)$ & $20.2(21.2 \%)$ & $21.5(24.3 \%)$ \\
\hline $95 \% \mathrm{CI}$ & [17.6-38.7\%] & {$[21.4-33.6 \%]$} & [11.6-31.8\%] & [16.4-32.3\%] \\
\hline Yes & $108.5(72.8 \%)$ & $102.4(72.5 \%)$ & $75.0(78.8 \%)$ & $67.0(75.7 \%)$ \\
\hline $95 \% \mathrm{CI}$ & {$[61.3-82.4 \%]$} & {$[66.4-78.6 \%]$} & {$[68.2-88.4 \%]$} & {$[67.7-83.6 \%]$} \\
\hline
\end{tabular}

Permanent discontinuation of treatment considered as failure

$D A S 28$-ESR disease activity score in 28 joints using the erythrocyte sedimentation rate, $L D A$ low disease activity

$(p=0.071)$ and 12 months $(p=0.555)$. Using the propensity score for adjustment on RA and patients characteristics, results were similar to the primary analysis: treatment maintenance at 6 months in switch and no-switch patients was observed in $86.1 \%$ (95\% CI [76.1-92.2\%]) and
92.8\% (95\% CI [88.4-95.6\%]) of patients, respectively. At 12 months, these respective proportions were $76.4 \%$ (95\% CI [65.3-84.4\%]) and $82.3 \%$ (95\% CI [76.3-86.8\%]). No significant difference was found between groups at 
Table 3 DAS28-ESR improvement or maintenance of remission/LDA status at 12 months

\begin{tabular}{|c|c|c|c|c|}
\hline & \multicolumn{2}{|c|}{ Main population $(n=314)$} & \multicolumn{2}{|c|}{ Stable population $(n=204)$} \\
\hline & $\overline{\text { Switch }(n=94)}$ & No-switch $(n=220)$ & $\overline{\text { Switch }(n=68)}$ & No-switch $(n=136)$ \\
\hline \multicolumn{5}{|c|}{ Without propensity score } \\
\hline$n$ & 91 & 204 & 67 & 128 \\
\hline Missing & 3 & 16 & 1 & 8 \\
\hline No & $28(30.8 \%)$ & $86(42.2 \%)$ & $20(29.9 \%)$ & $50(39.1 \%)$ \\
\hline $95 \% \mathrm{CI}$ & {$[21.5-41.3 \%]$} & {$[35.3-49.3 \%]$} & {$[19.3-42.3 \%]$} & {$[30.6-48.1 \%]$} \\
\hline Yes & $63(69.2 \%)$ & $118(57.8 \%)$ & $47(70.1 \%)$ & $78(60.9 \%)$ \\
\hline $95 \% \mathrm{CI}$ & {$[58.7-78.5 \%]$} & {$[50.8-64.7 \%]$} & {$[57.7-80.7 \%]$} & {$[51.9-69.4 \%]$} \\
\hline \multicolumn{5}{|c|}{ With propensity score } \\
\hline$n$ & 149.8 & 148.4 & 96.3 & 95.7 \\
\hline No & $45.5(30.4 \%)$ & $58.9(39.7 \%)$ & $29.7(31.2 \%)$ & $36.6(38.1 \%)$ \\
\hline $95 \% \mathrm{CI}$ & {$[19.5-41.7 \%]$} & {$[33.0-46.6 \%]$} & {$[19.8-43.5 \%]$} & {$[29.1-46.6 \%]$} \\
\hline Yes & $104.3(69.6 \%)$ & $89.5(60.3 \%)$ & $65.6(68.8 \%)$ & $59.6(61.9 \%)$ \\
\hline $95 \% \mathrm{CI}$ & {$[58.3-80.5 \%]$} & {$[53.4-67.0 \%]$} & {$[56.5-80.2 \%]$} & {$[53.4-70.9 \%]$} \\
\hline
\end{tabular}

Permanent discontinuation of treatment considered as failure, regardless if a DAS28 value was available within the predefined time window for 12 months

$D A S 28-E S R$ disease activity score in 28 joints using the erythrocyte sedimentation rate, $L D A$ low disease activity

6 months $\quad(p=0.102) \quad$ and $\quad 12$ months $(p=0.253)$.

In the stable population, using the propensity score, results were also close to the primary analysis: the rates of therapeutic maintenance at 6 months in switch and no-switch patients were $80.0 \%$ (95\% CI [67.3-88.2\%]) and 94.8\% (95\% CI [89.2-97.5\%]), respectively, with a statistical difference $(p=0.002)$. At 12 months, these respective rates were $73.3 \%(95 \% \mathrm{CI}$ $[60.1-82.8 \%])$ and $83.6 \% \quad(95 \% \quad C I$ [75.9-89.0\%]), without difference $(p=0.087)$.

\section{Predictive Factors of Switching}

In the main population, univariate (Table S3) analyses showed the following significant predictor parameters $(p$ value $\leq 0.20$ ) for the TCZ switch: at least one extra articular manifestation of RA $(p=0.084)$, rheumatoid nodules $(p=0.014)$, duration of IV TCZ before inclusion (in months, $p=0.004 ; \pm 2$ years, $p=0.052$ ), DAS28-ESR (mean value, $p<0.001$; three categories, $p=0.042 ; \pm 3.2, p<0.001)$ and DAS28CRP (mean value, $p<0.001$; three categories, $p=0.006)$.

After collinearity controls, three covariates entered the multivariate model and were kept after backward selection: rheumatoid nodules, duration of IV TCZ before inclusion (in months), and DAS28-ESR score $( \pm 3.2)$.

As an interaction was shown between the duration of IV TCZ before inclusion and the DAS28-ESR score $\quad(\mathrm{OR}=1.02, \quad 95 \% \quad \mathrm{CI}$ [1.00-1.05], $p=0.085)$, the multivariate analysis was stratified according to the DAS28-ESR score at inclusion. In the 208 patients with a DAS28-ESR score $\leq 3.2$ at inclusion, multivariate analysis (Table S4) showed no parameters significantly associated to the switch of TCZ (previous duration of IV TCZ: $p=0.267$; rheumatoid nodules: $p=0.112$ ). Conversely in 


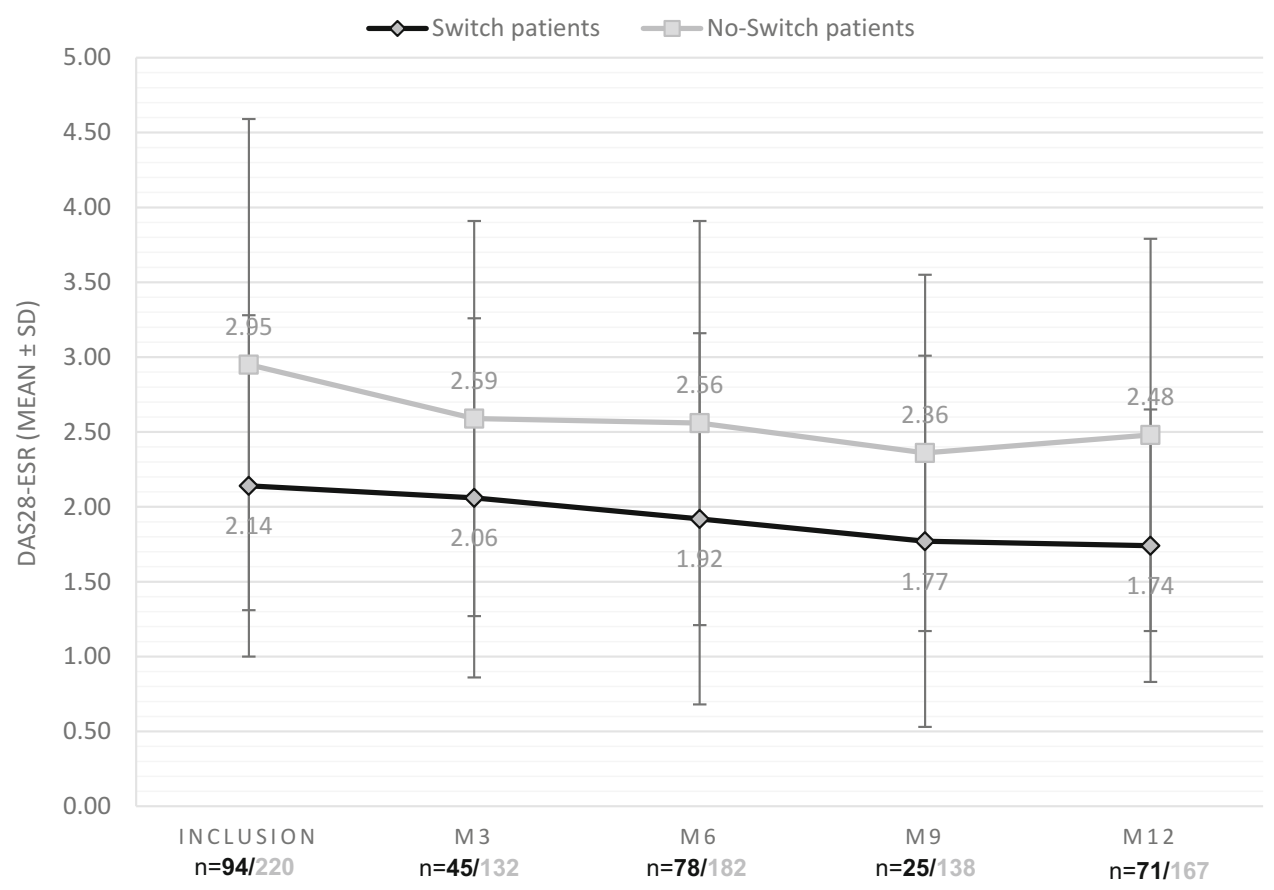

Fig. 2 DAS28-ESR evolution over the study period, according to switch and no-switch patients of the main population. $D A S 28-E S R$ disease activity score in 28 joints using the erythrocyte sedimentation rate

the 106 patients with a DAS28-ESR score $>3.2$ at inclusion, analysis showed that both parameters were significantly associated to the switch: rheumatoid nodules (OR $=4.78,95 \%$ CI [1.23-18.55], $p=0.024)$ and duration of IV TCZ before inclusion $(\mathrm{OR}=1.37, \quad 95 \% \quad \mathrm{CI}$ [1.08-1.73], $p=0.009$ ). It should be noted that only 17 switch patients were included in this analysis. This association was also demonstrated in the stable population (eight switch patients) but only with the duration of IV TCZ before inclusion $(\mathrm{OR}=2.05,95 \%$ CI $[1.16-3.60]$, $p=0.013)$.

\section{Patient Self-reported Questionnaire}

At inclusion, 197 patients (63\%) including 66 switch patients of the main population fulfilled this questionnaire that comprises questions on reasons for the potential switch of administration route of TCZ (from IV to SC). At the time of the questionnaire completion, $90.9 \%$ of the switch patients claimed to prefer switching to ambulatory SC injections of TCZ while noswitch patients were only $24.4 \%$ in this case.
Reasons to consider the SC route at inclusion were in descending order: constraint of a monthly visit (71.4\%), greater autonomy $(40.5 \%)$, health economy $(26.2 \%)$, difficulties/pain during IV (7.1\%), scared of hospital (2.4\%) (Fig. S1). Reasons to prefer staying in IV at inclusion were in descending order: fear of a less close follow-up (64.9\%), absence of medical attendance $(50.5 \%)$, fear of allergic adverse event (31.2\%), frequency of injections (30.9\%), possibility to socialize with other patients (23.6\%), fear of SC injection (11.8\%), other SC treatments (6.4\%) (Fig. S2).

At 6 months, 50/81 (62\%) patients still treated with SC TCZ completed the follow-up questionnaire. They perceived to be followed as well as if they were treated by the IV formulation of IV in $81.4 \%$ of the cases.

\section{Safety}

In the population of patients who changed from IV to SC formulation of TCZ, 21 patients stopped the treatment between baseline and 12 months: eight for adverse events (three 


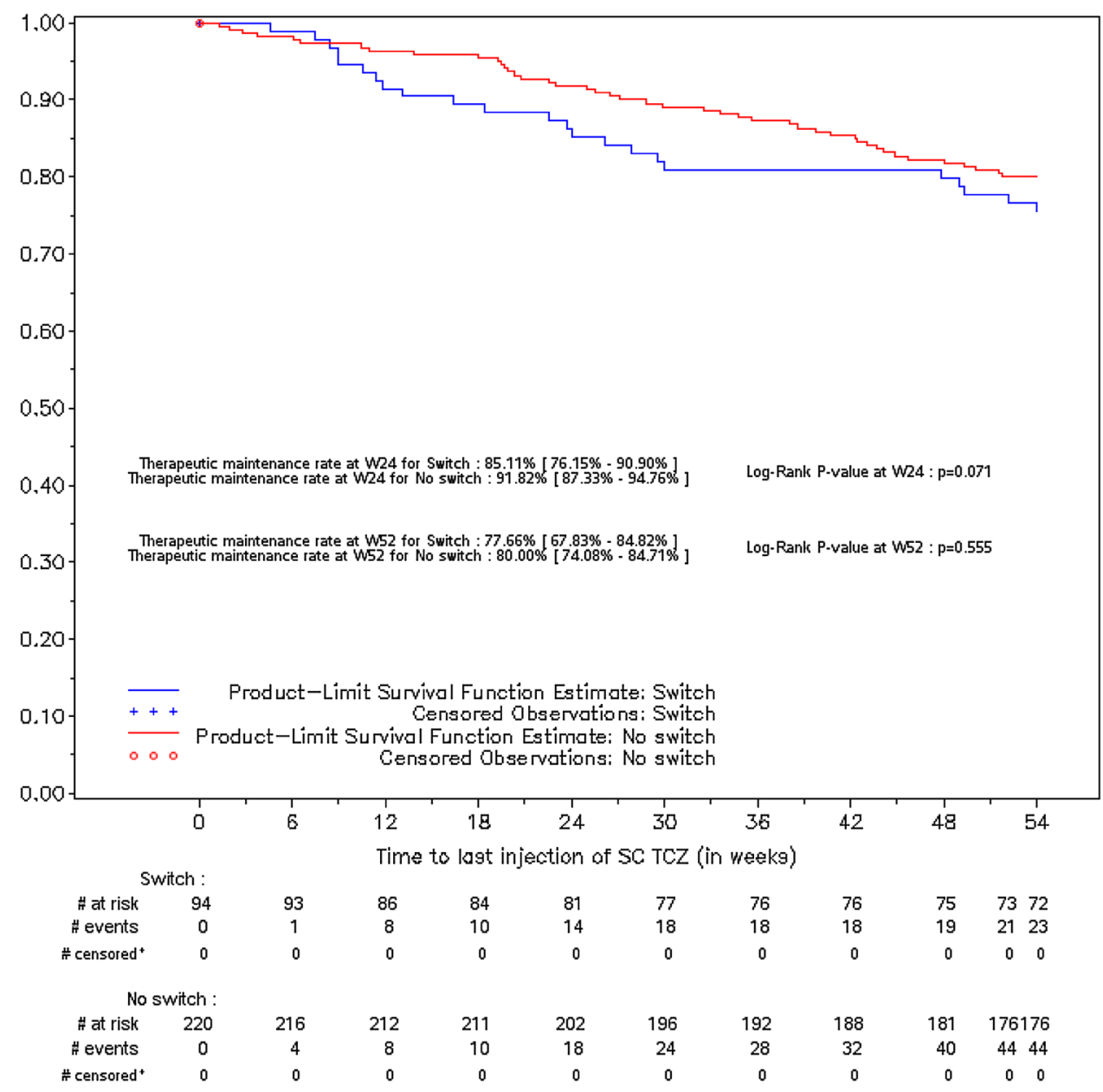

Fig. 3 Maintenance of TCZ treatment according to switch and no-switch patients. Kaplan-Meier curve of the main population. $S C$ subcutaneous, $T C Z$ tocilizumab

injection site reactions, four infections [one skin infection, one bronchopulmonary infection, one urinary tract infection, one biliary infection] and one liver cytolysis), eleven for inefficiency (three primary failures, eight secondary failures), and two data were missing.

In the population of patients who did not change from IV to SC tocilizumab, 44 patients stopped the treatment between baseline and 12 months: twenty for adverse events (ten infections [five skin infections, two bronchopulmonary, two urinary tract infections, one biliary infection], two mild allergies, two liver cytolysis, three cytopenia, one colorectal cancer, one high blood pressure, one vagal discomfort), thirteen for inefficiency (thirteen secondary failures), three for remission, four for other reasons (two switch to SC TCZ [outside the protocol], one multiple surgeries, one desire for parenthood) and four data were missing.

\section{DISCUSSION}

The RoSwitch study, a 1-year non-interventional secondary data use study, showed that after switching from IV to SC TCZ, seven out of ten patients maintained a remission/LDA status at 6 months. This proportion was comparable to what was observed in patients who continued the IV formulation. Since RoSwitch was a realworld study, and the switch was a consequence of a shared decision between the patient and the rheumatologist, without randomization, 
there were some differences between the two groups at inclusion. Therefore, a propensity score was used to balance the two groups, and no significant difference was finally found concerning the maintenance of effectiveness.

Similar results were found at 12 months, with even a favorable trend for the switch group. This favorable trend could be explained by the higher proportion of switch patients who already had a DAS28-ESR value $\leq 3.2$ at inclusion (81.9 vs. $59.5 \%$ in switch and no-switch patients, respectively). Effectively, in our study, RA appeared to be well controlled at inclusion. This initial RA control was also observed in switched patients for another biotherapy in a recent single-center Italian non-interventional study [12], conducted on 51 RA patients who switched to SC formulation from IV administration of abatacept: $78 \%$ of patients who maintained the SC formulation and $57 \%$ of patients who returned to IV infusions had DAS28 remission at SC formulation start.

These results seem to confirm in a real-world setting previous findings from interventional studies and especially from the SUMMACTA study [11], despite differences in patients characteristics (Table S5). The phase III SUMMACTA study has shown the non-inferiority of SC TCZ compared with IV TCZ at 6 months in patients with moderate-to-severe active RA. At 6 months, patients were re-randomized to one or the other formulation, and at 49 weeks the effectiveness and tolerance observed in patients who switched was comparable to those of patients who remained in their first TCZ formulation [13]. Similar results were obtained in the MUSASHI study [14] after an open-label switch, following by a double-blind comparative period of SC TCZ monotherapy versus IV TCZ monotherapy that showed the non-inferiority of SC administration [15].

Only two non-interventional studies were conducted, and both were in Japan. The first one [16] was a 3-month study in 58 RA patients for whom SC TCZ was initiated following IV TCZ. In this study, the mean value of DAS28ESR was not changed 3 months after the administration route was switched. However, it was a short-term study, with a limited number of patients and without analysis of patients remaining in IV. In the second study [17], the 219 patients included in the TCZ-IV-SC group had similar median CDAI scores and mean DAS28-ESR over 26 weeks.

Some differences were observed between switch and no-switch patients. Switch patients were younger with lower disease activity, which can suggest that since the switch was the consequence of a shared decision, hospital rheumatologists have probably encouraged older and more comorbid patients with active disease to keep a hospital follow-up.

In multivariate analysis, an association between the rheumatoid nodules, the duration of IV TCZ before inclusion, and the switch has been found in patients with an initial DAS28ESR $>3.2$. Association with rheumatoid nodules is difficult to interpret. However, the association between duration of IV TCZ before inclusion and the switch was found both in the main and the stable populations. It should be noted that only 17 switch patients of the main population (and eight from the stable population) were included in these analyses. It could be suggested that for these patients, for whom the rheumatologist did not consider it necessary to change the therapeutic class, a shared decision of a switch was chosen.

The BREVACTA [10] and MUSASHI [14] studies suggested that the effectiveness of TCZ could be reduced after the switch in some patients with high BMI. In the non-interventional study of Iwamoto [16], $\triangle \mathrm{DAS} 28$ tended to be worsened after switching in the high-bodyweight groups, although no statistical significance was found. In our analysis using data mining techniques, BMI was not significantly related to the DAS28-ESR change. However, since this is only an exploratory analysis, it seems reasonable to maintain a reluctance to switch in patients with high BMI.

This analysis was only based on baseline parameters available in the "RIC Nord de France" EMR database. In particular, patient attitudes toward safety and convenience, past experience, perception of current disease status, health literacy, and socioeconomic status might affect the decision for the route administration, as well as health care staff/institution-related factors (knowledge and experience, human 
resources) [18-20]. The fact that no factor was associated with the switch in patients in remission/LDA at inclusion could suggest that the decision to change treatment formulation is based on the patient's personal appreciation. Moreover, the patient self-reported questionnaire showed the importance of the a priori patient choice in the administration route because $90.9 \%$ of the switch patients claimed to prefer switching to ambulatory SC injections of TCZ while no-switch patients were only $24.4 \%$. Furthermore, switch patients highlighted the greater autonomy provided by SC injections before switching. Similar findings were observed in a survey identifying the determinants of patient preferences in the choice of anti-TNF therapies for RA, and the SC route was chosen for the convenience of treatment and in particular for home treatment [20].

We observed a high rate of treatment maintenance. By comparison, a similar rate was also observed 3 months after the switch from IV to SC formulation of abatacept in an Italian noninterventional study conducted in a small case series of RA patients [12]. In our study, although there was no statistically significant difference between the two groups in terms of therapeutic maintenance, a slight trend in favor to noswitch patients was observed. This could be explained mainly by injection site reactions in switch patients. It is a more common effect in patients treated by SC route as reported in SUMMACTA study [11].

At last, descriptive safety did not show any new signal, to the reserve that events were analyzed independently from the extracted database, which may have led to some bias.

Study limitations were mainly related to the use of data extracted from a preexisting database, which was built with objectives different from ours. The 314 patients of the main population were included by a limited number of rheumatologists located in one French area only. Consequently, the representativeness of our rheumatologist and patient populations cannot be ensured. Another limitation concerns study design, especially regarding the selection method and the conscious choice to define the dates of inclusion of patients based on availability of SC TCZ in France, which may have led to heterogenous exposition to treatments or RA characteristics between the two groups of patients. Additionally, some results should be interpreted with caution due to low number of patients or unbalanced subgroups of population.

The main strengths of our study were a longterm follow-up, a large real-world population with description of both switch and no-switch patients, using two kinds of population.

\section{CONCLUSIONS}

The RoSwitch study showed the maintenance of effectiveness at 6 and 12 months in RA patients switching from IV to SC TCZ. Similar effectiveness and therapeutic maintenance rates were observed for no-switch patients. No factor was associated with the switch in patients in remission/LDA at inclusion suggesting that patient's personal appreciation was preponderant in the choice of the switch.

\section{ACKNOWLEDGEMENTS}

The authors thank the patients who participated in the RoSwitch study.

Funding. This study was supported by Roche SAS and Chugai Pharma France, including data management, statistical analysis, and article processing charges. All authors had full access to all of the data in this study and take complete responsibility for the integrity of the data and accuracy of the data analysis.

Authorship. All named authors meet the International Committee of Medical Journal Editors (ICMJE) criteria for authorship for this article, take responsibility for the integrity of the work as a whole, and have given their approval for this version to be published.

Disclosures. Vincent Goëb received honoraria for punctual interventions for Roche Chugai. Xavier Deprez received honoraria for punctual interventions for Roche Chugai. Samuel Gally is an employee of Roche SAS. 
David Pau is an employee of Roche SAS. Isabelle Idier is an employee of Chugai Pharma France. René-Marc Flipo is a member of the national advisory committee of Roche Chugai. Jean Darloy, Nicolas Segaud, Jean-Hugues Salmon, Jean-Paul Eschard, Marie-Hélène Guyot, Eric Houvenagel, Nicolas Lecuyer, Laurent Marguerie, and Guy Baudens have nothing to disclose.

Compliance with Ethics Guidelines. In accordance with French law regarding non-interventional studies, the RoSwitch protocol was approved on April 7, 2016, by the Consultative Committee on Information Processing for Research in the Field of Health and was validated on August 1, 2016, by the Independent Administrative Authority Protecting Privacy and Personal Data. All patients were informed about the course of the study before enrolment. This study was performed in accordance with the Helsinki Declaration of 1964 and its later amendments.

Open Access. This article is distributed under the terms of the Creative Commons Attribution-NonCommercial 4.0 International License (http://creativecommons.org/licenses/ by-nc/4.0/), which permits any noncommercial use, distribution, and reproduction in any medium, provided you give appropriate credit to the original author(s) and the source, provide a link to the Creative Commons license, and indicate if changes were made.

\section{REFERENCES}

1. Guillemin F, Saraux A, Guggenbuhl P, et al. Prevalence of rheumatoid arthritis in France: 2001. Ann Rheum Dis. 2005;64(10):1427-30.

2. Gabriel SE, Crowson CS, Kremers HM, et al. Survival in rheumatoid arthritis: a population-based analysis of trends over 40 years. Arthritis Rheum. 2003;48:54-8.

3. Fautrel B, Gaujoux-Viala C. Aspects médico-économiques de la polyarthrite Rhumatoïde. Bull Acad Natle Méd. 2012;196(7):1295-306.
4. Srirangan S, Choy EH. The role of interleukine 6 in the pathophysiology of rheumatoid arthritis. Ther Adv Musculoskelet Dis. 2010;2(5):247-56.

5. Emery P, Keystone E, Tony HP, et al. IL-6 receptor inhibition with tocilizumab improves treatment outcomes in patients with rheumatoid arthritis refractory to anti-tumour necrosis factor biologicals: results from a 24-week multicentre randomised placebo-controlled trial. Ann Rheum Dis. 2008;67(11):1516-23.

6. Genovese MC, McKay JD, Nasonov EL, et al. Interleukin-6 receptor inhibition with tocilizumab reduces disease activity in rheumatoid arthritis with inadequate response to disease-modifying antirheumatic drugs: the tocilizumab in combination with traditional disease-modifying antirheumatic drug therapy study. Arthritis Rheum. 2008;58(10):2968-80.

7. Smolen JS, Beaulieu A, Rubbert-Roth A, et al. Effect of interleukin-6 receptor inhibition with tocilizumab in patients with rheumatoid arthritis (OPTION study): a double-blind, placebo-controlled, randomised trial. Lancet. 2008;371(9617):987-97.

8. Jones $\mathrm{G}$, Sebba A, Gu J, et al. Comparison of tocilizumab monotherapy versus methotrexate monotherapy in patients with moderate to severe rheumatoid arthritis: the AMBITION study. Ann Rheum Dis. 2010;69(1):88-96.

9. Fleischmann RM, Halland AM, Brzosko M, et al. Tocilizumab inhibits structural joint damage and improves physical function in patients with rheumatoid arthritis and inadequate responses to methotrexate: LITHE study 2-year results. J Rheumatol. 2013;40(2):113-26.

10. Kivitz A, Olech E, Borofsky M, et al. Subcutaneous tocilizumab vs placebo in combination with disease modifying antirheumatic drugs in patients with rheumatoid arthritis. Arthritis Care Res (Hoboken). 2014;66(11):1653-61.

11. Burmester GR, Rubbert-Roth A, Cantagrel A, et al. A randomised, double-blind, parallel-group study of the safety and efficacy of subcutaneous tocilizumab versus intravenous tocilizumab in combination with traditional disease-modifying antirheumatic drugs in patients with moderate to severe rheumatoid arthritis (SUMMACTA study). Ann Rheum Dis. 2014;73(1):69-74.

12. Reggia R, Franceschini F, Tincani A, et al. Switching from intravenous to subcutaneous formulation of abatacept: a single-center Italian experience on efficacy and safety. J Rheumatol. 2015;42:193-5.

13. Burmester GR, Rubbert-Roth A, Cantagrel A, et al. Efficacy and safety of subcutaneous tocilizumab 
versus intravenous tocilizumab in combination with traditional DMARDs in patients with RA at week 97 (SUMMACTA study). Ann Rheum Dis. 2016;75(1):68-74.

14. Ogata A, Atsumi T, Fukuda T, et al. Sustainable efficacy of switching from intravenous to subcutaneous tocilizumab monotherapy in patients with rheumatoid arthritis. Arthritis Care Res (Hoboken). 2015;67(10):1354-62.

15. Ogata A, Tanimura K, Sugimoto T, et al. Phase III study of the efficacy and safety of subcutaneous versus intravenous tocilizumab monotherapy in patients with rheumatoid arthritis. Arthritis Care Res (Hoboken). 2014;66(3):344-54.

16. Iwamoto N, Fukui S, Umeda M, et al. Evaluation of switching from intravenous to subcutaneous formulation of tocilizumab in patients with rheumatoid arthritis. Mod Rheumatol. 2016;26(5):662-6.
17. Atsumi T, Fujio K, Yamaoka K, et al. Safety and effectiveness of subcutaneous tocilizumab in patients with rheumatoid arthritis in a real-world clinical setting. Mod Rheumatol. 2018;28(5):780-8.

18. Desplats M, Pascart T, Jelin G, et al. Are abatacept and tocilizumab intravenous users willing to switch for the subcutaneous route of administration? A questionnaire-based study. Clin Rheumatol. 2017;36:1395-400.

19. Jin JF, Zhu LL, Chen M, et al. The optimal choice of medication administration route regarding intravenous, intramuscular, and subcutaneous injection. Patient Prefer Adherence. 2015;9:923-42.

20. Scarpato S, Antivalle M, Favalli EG, et al. Patient preferences in the choice of anti-TNF therapies in rheumatoid arthritis. Results from a questionnaire survey (RIVIERA study). Rheumatology (Oxford). 2010;49(2):289-94. 